\section{Homicídios masculinos em duas regiões brasileiras: análise do efeito da idade, período e coorte}

\author{
Male homicides in two regions of Brazil: analysis \\ of the effect of age, period, and cohort
}

\section{Homicidios masculinos en dos regiones brasileñas: análisis del efecto de la edad, período y cohorte}

\section{Resumo}

O objetivo foi avaliar o efeito da idade, período e coorte de nascimento na evolução temporal da mortalidade por homicídios em homens nos estados das regiões Nordeste e Sudeste do Brasil, entre o período de 1980 a 2014. Estudo ecológico de tendência temporal. Os modelos APC foram estimados usando funções estimáveis na inferência dos parâmetros. Os dados de mortalidade e os dados populacionais foram obtidos junto ao Departamento de Informática do SUS. As taxas de mortalidade por homicídio, segundo estados, foram padronizadas pelo método direto, tendo, como população padrão, a mundial, proposta pela Organização Mundial da Saúde. A Região Nordeste apresentou 317.711 óbitos por homicídios, e o Sudeste, 544.640 óbitos, correspondendo, respectivamente, a uma taxa média padronizada por 100.000 homens de 58,68 óbitos e 64,68 óbitos. As maiores taxas de mortalidade médias padronizadas foram observadas nos estados de Alagoas (157,74 óbitos) e Pernambuco (109,58 óbitos). Em todos os estados, observou-se aumento da mortalidade até a terceira década de vida, com redução progressiva para as demais faixas etárias. Verificou-se tendência ascendente para todos os estados da Região Nordeste, Minas Gerais e Espírito Santo; nos demais estados, houve descendência das taxas. Em todos os estados, observou-se aumento do risco de óbito nas gerações mais jovens, com exceção do Estado de São Paulo que mostrou perfil inverso. Os achados do presente estudo podem correlacionar-se com o processo de descontinuidade de coorte, no qual integrantes de coortes largas encontram menor oportunidade de acesso a emprego, renda e educação, aumentando o risco de envolvimento com a criminalidade e morte por homicídios.

Homicídio; Homens; Efeito Idade; Efeito Período; Efeito de Coortes
Laiane Felix Borges 1

Edinilsa Ramos de Souza 2

Adalgisa Peixoto Ribeiro 3

Glauber Weder dos Santos Silva 4

Cosme Marcelo Furtado Passos da Silva 2

Juliano dos Santos 5

Karina Cardoso Meira 1

doi: 10.1590/0102-311X00008719

\section{Correspondência}

K. C. Meira

Av. Senador Salgado Filho 2990, apto. 804, Natal, RN 59075-000, Brasil.

ninameira87@gmail.com

1 Escola de Saúde, Universidade Federal do Rio Grande do Norte, Natal, Brasil.

2 Escola Nacional de Saúde Pública Sergio Arouca, Fundação Oswaldo Cruz, Rio de Janeiro, Brasil.

3 Faculdade de Medicina, Universidade Federal de Minas Gerais, Belo Horizonte, Brasil.

4 Departamento de Enfermagem, Universidade Federal do Rio Grande do Norte, Natal, Brasil.

5 Instituto Nacional de Câncer José Alencar Gomes da Silva, Rio de Janeiro, Brasil. 


\section{Introdução}

Estudos sobre homicídio o consideram como expressão máxima da violência interpessoal e indicador universal da violência social ${ }^{1}$. Pesquisas têm evidenciado padrão de maior vulnerabilidade de pessoas que vivem em localidades com precárias condições de infraestrutura e equipamentos sociais, sobretudo, quando se analisam dados em diferentes escalas - regional, municipal e local 2,3,4,5,6,7,8,9,10. Outro padrão também recorrente se refere às características das vítimas dos homicídios: no Brasil e no mundo, são prioritariamente jovens, do sexo masculino, de raça/cor preta ou parda, com baixa escolaridade, pouca qualificação profissional, moradores de áreas periféricas e empobrecidas 11,12. Além disso, destacam-se as armas de fogo como meios mais comumente usados para concretizar a violência fatal 12,13 .

Os homicídios disseminaram-se pelo Brasil e tornaram-se problema substancial para as Unidades Federativas que, em décadas anteriores, apresentavam taxas de mortalidade baixas e, assim, não estavam preparadas para atuar na intensificação da violência ocorrida na primeira década dos anos 2000. Essa disseminação, no sentido da interiorização dos homicídios, foi marcada pela redução e estagnação das taxas nas capitais e regiões metropolitanas, sobretudo, nos estados de São Paulo e Rio de Janeiro, e aumento nos demais municípios 8,10. Nesse contexto, a Região Nordeste experimentou aumento de $80 \%$ nas taxas de mortalidade por homicídios, comparando-se os anos de 2004 e 20149.

Assim, este estudo pretendeu analisar o efeito da idade, do período e coorte de nascimento da mortalidade por homicídios masculinos, de 1980 a 2014, nos estados das regiões Nordeste e Sudeste.

O efeito idade representa as mudanças nas taxas de mortalidade, ou incidência por doenças ou agravos à saúde, correlacionadas aos processos biológicos, psicológicos e/ou sociais dos distintos grupos etários 14,15,16. O risco de envolvimento com atos criminosos não é uniforme durante a vida de uma pessoa, observa-se processo de iniciação nos primeiros anos de pré-adolescência e adolescência (12-13 anos), que atinge pico de ocorrência em torno dos 16 ou 17 anos e finaliza a partir da terceira década de vida 17,18 .

$\mathrm{O}$ efeito de período ou conjuntural refere-se às mudanças na mortalidade, devido aos eventos que ocorreram em determinados períodos e influenciam simultaneamente todas as faixas etárias, como mudanças na qualidade e cobertura dos registros de óbito, políticas públicas de transferência de renda ou de ampliação do acesso à educação profissional e ao emprego, maior circulação de armas de fogo, entre outros 19,20,21,22.

O efeito coorte ou estrutural é provocado por fatores que afetam uma geração e promovem mudanças de diferentes magnitudes nos coeficientes de mortalidade em sucessivos grupos etários, expressando a interação entre o efeito da idade e período como resultado do acúmulo de exposição ao longo da vida 19,20,21,22. Assim, a estrutura etária da população, associada ao efeito de período dos fatores criminogênicos, interfere no risco de morte por homicídios em jovens, sobretudo, os que integram coortes largas (em frequência relativa ou absoluta), promovendo o que é conhecido como descontinuidade de coorte, impactando nos salários relativos, na demanda por educação, nas oportunidades de emprego, nas taxas de desemprego e criminalidade 19,20,21,22.

\section{Metodologia}

Estudo ecológico de tendência temporal dos homicídios em pessoas do sexo masculino, nos estados das regiões Nordeste e Sudeste do Brasil, de 1980 a 2014. Os dados oficiais de mortalidade por homicídio foram obtidos no Sistema de Informações sobre Mortalidade (SIM), do Ministério da Saúde. Os registros de óbitos referentes à agressão foram os códigos E960 a E969, da 9a Revisão, e os códigos X85 a Y09, da 10a Revisão da Classificação Estatística Internacional das Doenças e Problemas Relacionados à Saúde (CID-9 e CID-10).

Os dados populacionais foram obtidos junto ao Departamento de Informática do SUS (DATASUS), com base nos Censos Populacionais de 1980, 1991, 2000 e 2010. As projeções populacionais para os anos intercensitários, em 1o de julho, foram as estimadas pelo Instituto Brasileiro de Geografia e Estatística (IBGE). Calcularam-se taxas específicas por faixa etária, por ano e padronizadas pelo método 
direto, tendo, como população padrão, a população mundial, proposta pela Organização Mundial da Saúde (OMS) 23.

As faixas etárias foram agrupadas em 13 grupos de cinco anos cada, a partir de 15 a 19 anos até 75 e mais anos. Os períodos também foram agrupados em sete períodos, com intervalos de cinco anos cada (1980-1984, 1985-1989, 1990-1994, 1995-1999, 2000-2004, 2005-2009 e 2010-2014). Finalmente, as coortes de nascimento iniciaram em 1905 e terminaram em 1999, perfazendo 19 coortes de nascimento.

A análise de tendência temporal foi realizada em duas etapas: inicialmente, realizou-se a análise exploratória, por meio da função de autocorrelação e do teste de Durbin-Watson; confirmada a dependência temporal dos dados, analisou-se a tendência temporal, por meio da regressão binomial negativa, pois os dados mostraram superdispersão (variância maior que a média). O número de óbitos esperados para cada ano representou a variável dependente; e o ano calendário centralizado, a variável independente, estimando-se modelos de regressão ponderados, proporcionais ao tamanho populacional, adicionando parâmetro conhecido como offset que consistiu no logaritmo da população de cada localidade em estudo 11,13. A tendência foi classificada em estacionária, decrescente ou ascendente, de acordo com o valor do risco relativo (RR) e respectivo intervalo de 95\% de confiança (IC95\%).

Analisou-se o efeito idade-período-coorte de nascimento (APC) para mortalidade por homicídios para os estados das regiões Nordeste e Sudeste, considerando-se distribuição de Poisson para o número de óbitos e que os efeitos atuam de maneira multiplicativa sobre a taxa. O logaritmo do valor esperado da taxa constitui função linear do efeito da idade, do período e da coorte 14,15,16:

$$
\ln \left(E\left[r_{\mathrm{ij}}\right]\right)=\ln \left(\frac{\theta_{\mathrm{ij}}}{N_{\mathrm{ij}}}\right)=\mu+\alpha_{i}+\beta_{j}+\gamma_{k}
$$

em que $E\left[r_{i j}\right]$ representa a taxa de mortalidade esperada na idade i e período j; $\theta_{\mathrm{ij}}$ o número de óbitos na idade i e período $\mathrm{j} ; \mathrm{N}_{\mathrm{ij}}$ denota a população sob risco de morte na idade i e período j; $\mu$ representa a média do efeito; $\alpha_{\mathrm{i}}$ corresponde ao efeito do grupo idade $\mathrm{i} ; \beta_{\mathrm{j}}$ o efeito do período $\mathrm{j}$ e; $\gamma_{\mathrm{k}}$ o efeito da coorte $\mathrm{k}$.

A estimação do modelo completo APC tem como principal limitação a relação linear entre os efeitos temporais (idade-período e coorte de nascimento), gerando o que se denomina no identifiability problem. Não há consenso na literatura sobre a melhor metodologia a ser utilizada para corrigir esse contrapondo, assim, neste trabalho, estimaram-se os parâmetros do efeito APC, por meio das funções estimáveis 14,15,16. Optou-se por manter como período de referência 1995-1999, pois o processo de interiorização e disseminação da violência iniciou-se a partir dos anos 2000 8, e como coorte de referência a de 1950-1954, pois coortes centrais tendem a ser mais estáveis e são mais completas que as primeiras e as últimas coortes 14,15,16. Ademais, decidiu-se trabalhar com faixas etárias e períodos com mesmo intervalo para evitar a ocorrência de novo problema de identificabilidade, decorrente de padrões cíclicos artificiais, quando se utilizam períodos e faixas etárias de tamanhos distintos 14,15,16.

O ajuste dos modelos aos dados foi verificado por meio da estatística deviance, considerandose estatisticamente significativos os resultados com $\mathrm{p} \leq 0,05$ 14,15,16. As análises para estimação do modelo APC foram realizadas por meio da biblioteca Epi 1.1.18, do programa R, versão 3.2.1 (http:// www.r-project.org).

Destaca-se, também, que foi avaliado o efeito de período para os registros de óbitos classificados como evento cuja intenção é indeterminada (ECI), correspondendo à codificação E980 a E989 (CID-9) e Y10 a Y34 (CID-10), pois melhorias na qualidade da informação podem promover efeito de período na evolução temporal da mortalidade 14,15,16.

\section{Resultados}

No período estudado, a Região Nordeste apresentou 317.711 óbitos de pessoas do sexo masculino por homicídios, e, o Sudeste, 544.640, correspondendo, respectivamente, à taxa média padronizada por 100 mil homens de 58,68 óbitos e 64,68 óbitos. As maiores taxas de mortalidade médias padronizadas foram observadas nos estados de Alagoas (157,74 óbitos), Pernambuco (109,58 óbitos), Espírito Santo (104,60 óbitos) e Rio de Janeiro (93,97 óbitos). 
Nos estados pesquisados, observaram-se taxas de mortalidade de maior magnitude nas faixas etárias de 20-24 e 25-29 anos, com redução progressiva com o avançar da idade (Figuras 1a e 1b). Nos estados da Região Nordeste, verificou-se perfil ascendente nas taxas médias padronizadas de mortalidade, mostrando maior magnitude no quinquênio de 2010-2014, com exceção de Pernambuco, com maior coeficiente no período de 2000-2004 e posterior redução (Figura 1c). Os estados de Minas Gerais e Espírito Santo apresentaram aumento dos coeficientes nos dois primeiros quinquênios dos anos 2000, com posterior redução, enquanto que, em São Paulo e no Rio de Janeiro, observou-se descendência nas taxas a partir dos anos 2000 (Figura 1d).

A evolução da mortalidade, segundo coorte, evidencia aumento dos coeficientes para gerações de homens mais jovens, exceto nos estados de Pernambuco, Rio de Janeiro, São Paulo e Minas Gerais, que mostram redução dos coeficientes para homens nascidos a partir da década de 1980 (Figuras 1e e 1f).

A análise da mortalidade por homicídios em homens mostrou tendência ascendente em todos os estados do Nordeste e nos estados do Espírito Santo e Minas Gerais, e, descendente nos estados de São Paulo e Rio de Janeiro (Tabela 1). Destaca-se que, na Região Nordeste, verificou-se tendência ascendente para todas as faixas etárias estudadas, e, no Sudeste, perfil inverso para todos os grupos etários a partir dos 15-19 anos (Tabela1).

O resultado dos modelos APC mostrou que, em todos os estados da Região Nordeste, assim como na região como um todo, os modelos idade-coorte (AC) e idade-período (AP) se ajustaram melhor aos dados, quando comparados ao modelo apenas com a idade e o modelo idade-drift. $\mathrm{O}$ modelo completo foi significativamente melhor do que o com dois fatores $\operatorname{AP}(\mathrm{p}<0,0001)$ e AC $(\mathrm{p}<0,0001)$ para os estados analisados, com exceção do Estado da Bahia, no qual o modelo que melhor se ajustou aos dados foi o idade-coorte (AC).

O efeito da idade ajustado, estimado em modelos probabilísticos, evidencia aumento progressivo do risco de morte a partir da faixa etária de 15-19 anos, atingindo maior valor nas faixas etárias da

\section{Figura 1}

Taxa de mortalidade média por homicídios em 100 mil homens nos estados das regiões Nordeste e Sudeste, Brasil, no período de 1980 a 2014.

1a) Nordeste (idade)

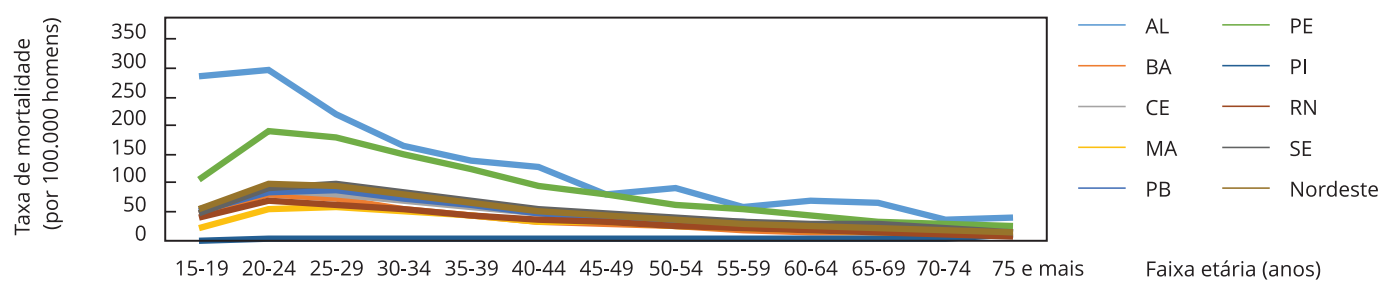

1b) Sudeste (idade)

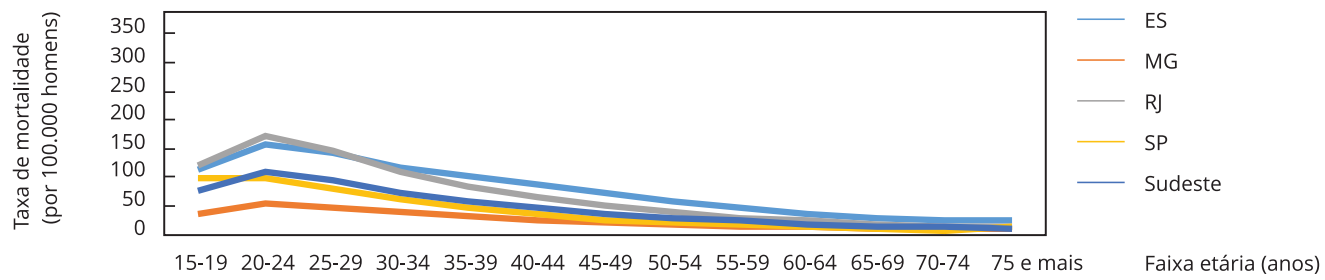

(continua) 


\section{Figura 1 (continuação)}

1c) Nordeste (período)
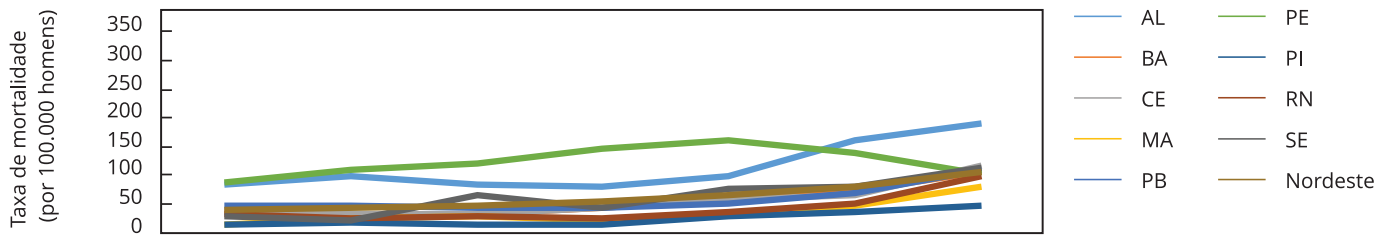

1980-1984 1985-1989 1990-1994 1995-1999 2000-2004 2005-2009 2010-2014 Período

1d) Sudeste (período)

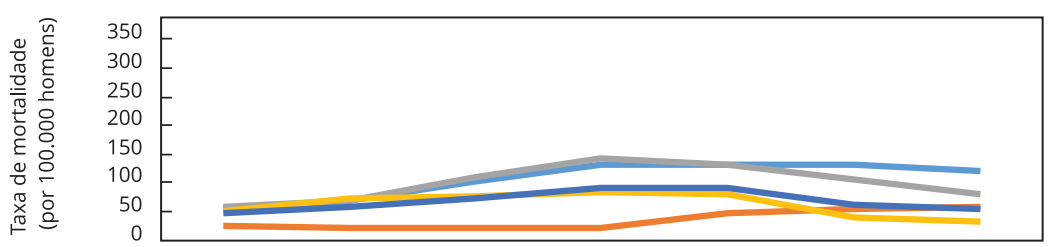

ES

- MG

RJ

- SP

- Sudeste

1980-1984 1985-1989 1990-1994 1995-1999 2000-2004 2005-2009 2010-2014 Período

1e) Nordeste (coorte)

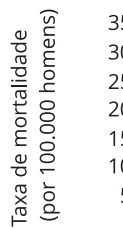

$\begin{array}{r}350 \\ 300 \\ 250 \\ 200 \\ 150 \\ 100 \\ 50 \\ 0\end{array}-$

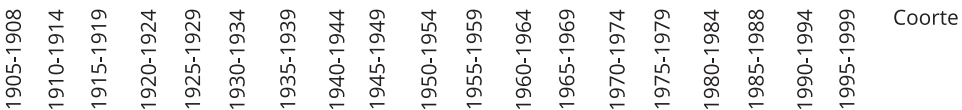

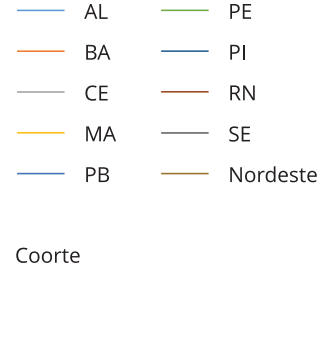

1f) Sudeste (coorte)
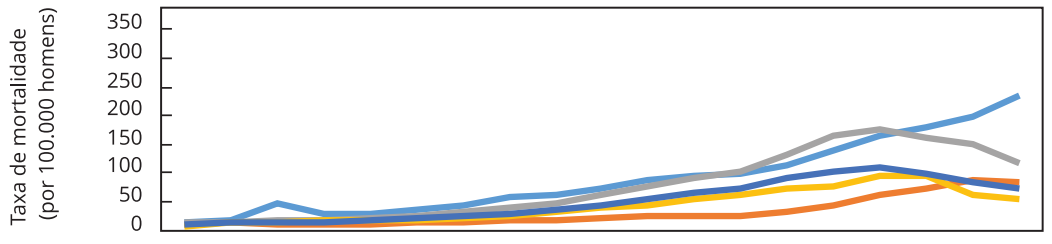

MG

$-\mathrm{RJ}$

- SP

Sudeste

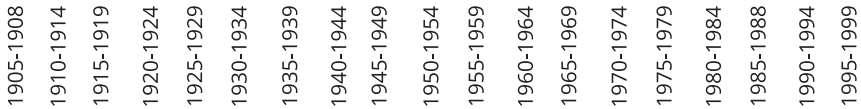

Coorte

Estados: $\mathrm{AL}=$ Alagoas; $\mathrm{BA}=$ Bahia; $\mathrm{CE}=$ Ceará; $\mathrm{ES}=$ Espírito Santo; MA = Maranhão; MG = Minas Gerais; PB = Paraíba; $\mathrm{PE}=$ Pernambuco; PI = Piauí; RJ = Rio de Janeiro; RN = Rio Grande do Norte; SE = Sergipe; SP = São Paulo. 


\section{Tabela 1}

Tendência temporal da mortalidade por homicídios nos estados das regiões Nordeste e Sudeste, Brasil, por meio de regressão binomial negativa, no período de 1980 a 2014.

\begin{tabular}{|c|c|c|c|}
\hline & \multirow[t]{2}{*}{$\mathbf{R R}$} & \multicolumn{2}{|c|}{ IC95\% } \\
\hline & & Limite inferior & Limite superior \\
\hline \multicolumn{4}{|l|}{ Nordeste } \\
\hline \multicolumn{4}{|l|}{ Faixa etária (anos) } \\
\hline $15-19$ & 1,070 & 1,069 & 1,071 \\
\hline $20-24$ & 1,043 & 1,043 & 1,044 \\
\hline $25-29$ & 1,032 & 1,031 & 1,033 \\
\hline $30-34$ & 1,024 & 1,023 & 1,025 \\
\hline $35-39$ & 1,019 & 1,017 & 1,020 \\
\hline $40-44$ & 1,018 & 1,016 & 1,019 \\
\hline $45-49$ & 1,012 & 1,011 & 1,014 \\
\hline $50-54$ & 1,013 & 1,011 & 1,015 \\
\hline $55-59$ & 1,009 & 1,006 & 1,011 \\
\hline $60-64$ & 1,011 & 1,008 & 1,014 \\
\hline $65-69$ & 1,015 & 1,011 & 1,018 \\
\hline $70-74$ & 1,013 & 1,008 & 1,017 \\
\hline $75 e+$ & 1,017 & 1,013 & 1,021 \\
\hline Total & 1,033 & 1,032 & 1,033 \\
\hline \multicolumn{4}{|l|}{ Estados } \\
\hline Alagoas & 1,035 & 1,034 & 1,036 \\
\hline Bahia & 1,058 & 1,057 & 1,059 \\
\hline Ceará & 1,051 & 1,050 & 1,052 \\
\hline Maranhão & 1,056 & 1,054 & 1,058 \\
\hline Paraíba & 1,036 & 1,035 & 1,038 \\
\hline Pernambuco & 1,003 & 1,003 & 1,004 \\
\hline Piauí & 1,050 & 1,048 & 1,053 \\
\hline Rio Grande do Norte & 1,056 & 1,054 & 1,058 \\
\hline Sergipe & 1,038 & 1,036 & 1,040 \\
\hline \multicolumn{4}{|l|}{ Sudeste } \\
\hline \multicolumn{4}{|l|}{ Faixa etária (anos) } \\
\hline $15-19$ & 1,062 & 1,056 & 1,068 \\
\hline $20-24$ & 1,048 & 1,043 & 1,052 \\
\hline $25-29$ & 1,040 & 1,036 & 1,045 \\
\hline $30-34$ & 1,039 & 1,034 & 1,045 \\
\hline $35-39$ & 1,034 & 1,028 & 1,040 \\
\hline $40-44$ & 1,027 & 1,020 & 1,034 \\
\hline $45-49$ & 1,008 & 1,000 & 1,016 \\
\hline $50-54$ & 1,013 & 1,004 & 1,023 \\
\hline $55-59$ & 1,010 & 0,998 & 1,023 \\
\hline $60-64$ & 1,034 & 1,018 & 1,050 \\
\hline $65-69$ & 1,019 & 1,002 & 1,036 \\
\hline $70-74$ & 0,995 & 0,974 & 1,016 \\
\hline $75 e+$ & 1,042 & 1,040 & 1,044 \\
\hline Total & 1,038 & 1,036 & 1,040 \\
\hline \multicolumn{4}{|l|}{ Estados } \\
\hline Espírito Santo & 1,011 & 1,010 & 1,012 \\
\hline Minas Gerais & 1,042 & 1,041 & 1,043 \\
\hline Rio de Janeiro & 0,993 & 0,993 & 0,994 \\
\hline São Paulo & 0,960 & 0,960 & 0,961 \\
\hline
\end{tabular}

IC95\%: intervalo de 95\% de confiança; RR: risco relativo. 
terceira década da vida, com posterior redução. Na Região Nordeste, as maiores taxas ajustadas são observadas nos estados de Pernambuco e Alagoas; e, no Sudeste, no Espírito Santo e Rio de Janeiro (Figuras 2 e 3).

O efeito do período após estimação do modelo APC, ajustado pelo efeito da idade e coorte, apontou aumento do risco de morte, estatisticamente significativo, em relação ao período de referência (1995-1999) para todos os demais quinquênios nos estados de Alagoas, Ceará e Rio Grande do Norte, padrão semelhante foi verificado no Piauí, exceto para o período de 1990 a 1994 (RR = 0,92; IC95\%: 0,89-0,95), e nos estados do Maranhão e Paraíba, com exceção do período de 2000 a 2004, no qual o risco de morte não foi estatisticamente significativo, sendo, respectivamente, RR = 1,001 (IC95\%: 0,98-1,03) e RR = 0,99 (IC95\%: 0,97-1,02) (Figura 3). Destaca-se que, nos estados do Ceará, Maranhão, Paraíba e Rio Grande do Norte, o período de 2010-2014 apresentou o maior risco de morte em relação ao período de referência.

Em contrapartida, em toda a Região Nordeste e no Estado de Sergipe, houve efeito protetor ( $R R<$ 1) para todos os períodos em relação ao período de referência, perfil semelhante foi apresentado por Pernambuco, que evidenciou efeito de proteção em relação ao período de 1995 a 1999, com exceção do período de 2000 a 2004 que mostrou aumento do risco de morte (RR = 1,04; IC95\%: 1,01-1,06) (Figura 3). Ainda, no Estado da Bahia, observou-se risco de óbito não estatisticamente significativo em todos os períodos, em relação ao período de referência (Figura 3). Na Região Sudeste, para os estados do Espírito Santo e de Minas Gerais, verificou-se efeito de proteção até o período de 19851989, aumento na década de 1990, que se manteve até o início dos anos 2000 (2000-2004), e redução no último quinquênio. Em contrapartida, em São Paulo e no Rio de Janeiro, verificou-se aumento do

\section{Figura 2}

Resultados do efeito da idade, período e coorte ajustados para a mortalidade por homicídios em homens nos estados da Região Nordeste, Brasil, 1980 a 2014.

2a) Coorte/Período

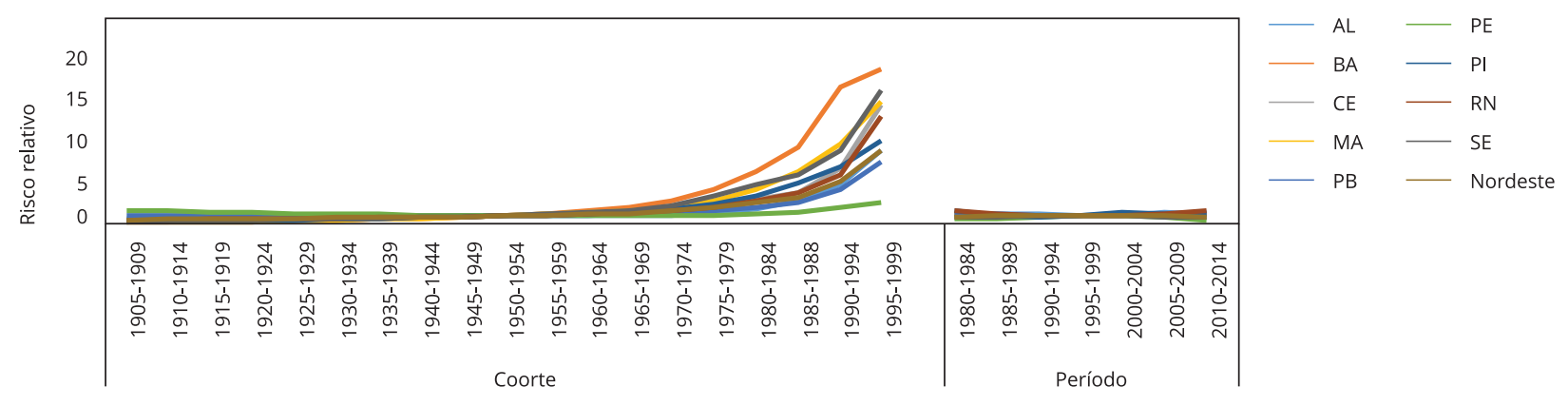

2b) Idade

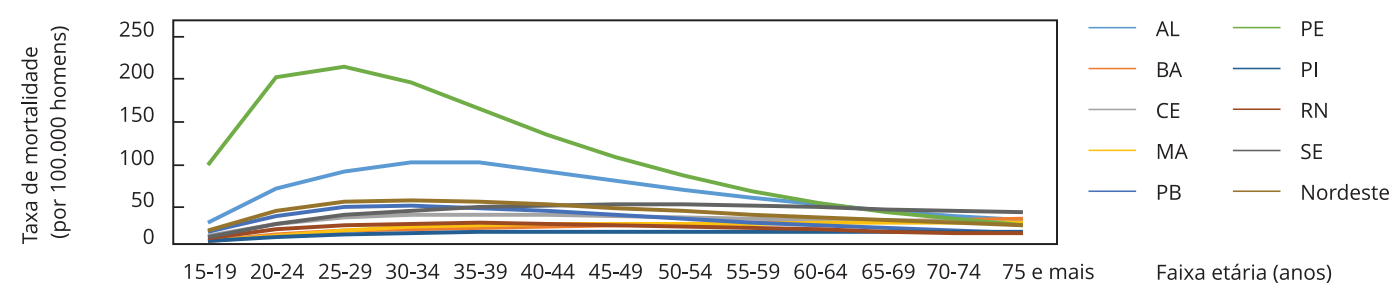

Estados: AL = Alagoas; BA = Bahia; CE = Ceará; MA = Maranhão; PB = Paraíba; PE = Pernambuco; PI = Piauí; RN = Rio Grande do Norte; SE = Sergipe. 
Figura 3

Resultados do efeito da idade, período e coorte ajustados para a mortalidade por homicídios em homens nos estados da Região Sudeste, Brasil, no período de 1980 a 2014.

3a) Coorte/Período

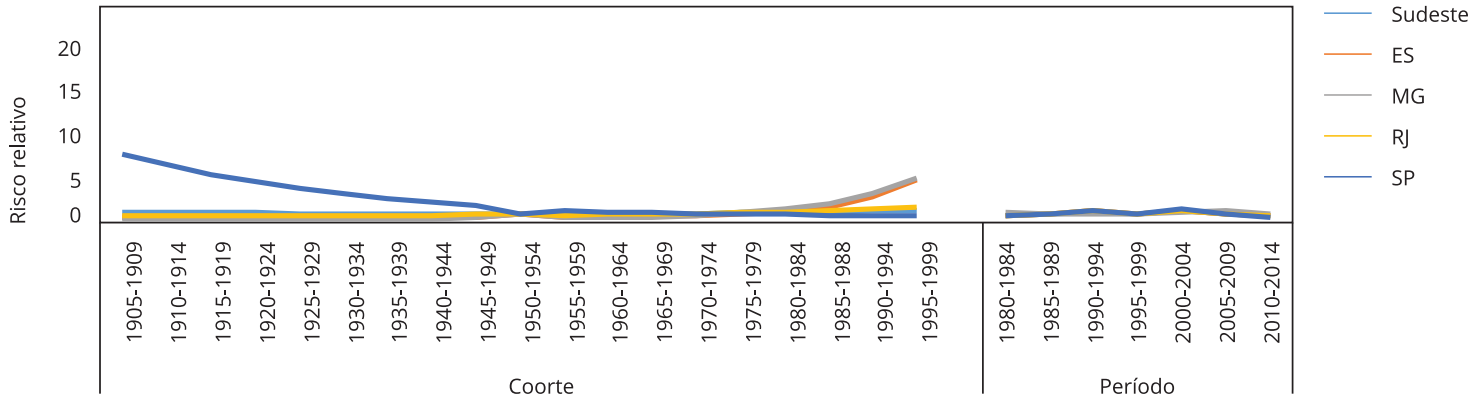

3b) Idade

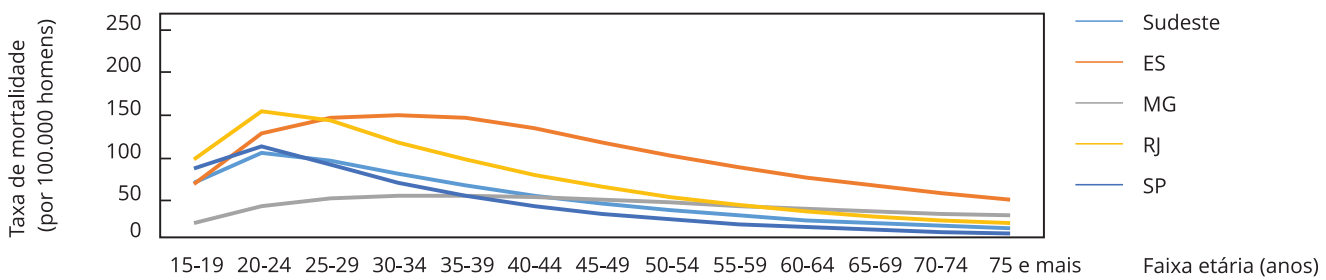

Estados: ES = Espírito Santo; MG = Minas Gerais; RJ = Rio de Janeiro; SP = São Paulo.

risco de morte na década de 1990 até o primeiro quinquênio dos anos 2000, com efeito de proteção para os demais quinquênios (Figura 4).

Merece destaque o efeito de período dos registros de óbito classificados como ECI, observado nos estados de Pernambuco, Espírito Santo, Rio de Janeiro e Minas Gerais. Em Pernambuco, houve aumento do risco de óbitos classificados como ECI, em todos os períodos, comparado ao período de referência (1995-1997) e redução do risco de morte por homicídios em todos os períodos (Figura 4). Verificou-se perfil semelhante no Espírito Santo na década de 1980 e período de 2010-2014, Minas Gerais, nos períodos de 1985-1989, 1990-1994 e 2010-2014, assim como no Rio de Janeiro, no período de 2005 a 2014 (Figura 4).

O efeito da coorte de nascimento, após o ajuste pelo efeito da idade e período do óbito, indicou efeito de proteção $(R R<1)$ nas coortes anteriores às de referência (1950-1954), havendo aumento progressivo do risco de morte por homicídios para homens nascidos após a coorte de 1960-1964, e o maior risco foi verificado nas coortes de nascimento mais jovem (1995-1999), sendo o risco mais elevado para homens residentes nos estados da Bahia, Ceará, Maranhão, Sergipe e Rio Grande do Norte (Figura 3). Esse perfil foi observado em todos os estados, com exceção de Pernambuco, em que houve aumento do risco de morte para as coortes mais velhas, quando comparadas às de referência com redução progressiva até a coorte de 1945-1949, mostrando efeito de proteção até a geração de 1970-1974, momento a partir do qual o risco de morte por homicídio aumentou (Figura 3).

Na Região Sudeste, assim como nos estados de Minas Gerais e do Rio de Janeiro, observou-se redução do risco de morte por homicídios para homens nascidos até a década de 1960, com elevação do risco estatisticamente significativa para as coortes a partir de 1975-1979; no Espírito Santo, o padrão foi similar, no entanto, houve aumento do risco para as coortes a partir da década de 1980 


\section{Figura 4}

Resultados do efeito de período ajustado pelo efeito da idade e coorte de nascimento das taxas de mortalidade classificadas como evento cuja intenção é indeterminada, nos estados da regiões Nordeste e Sudeste, Brasil, no período de 1980 a 2014.

4a) Nordeste

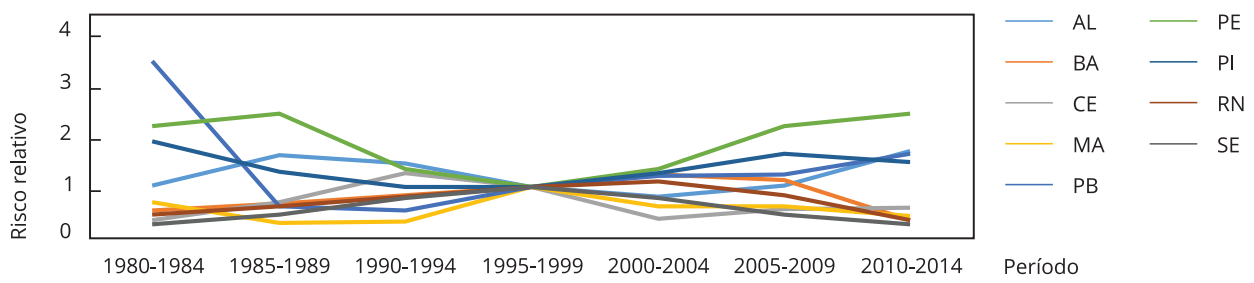

4b) Sudeste

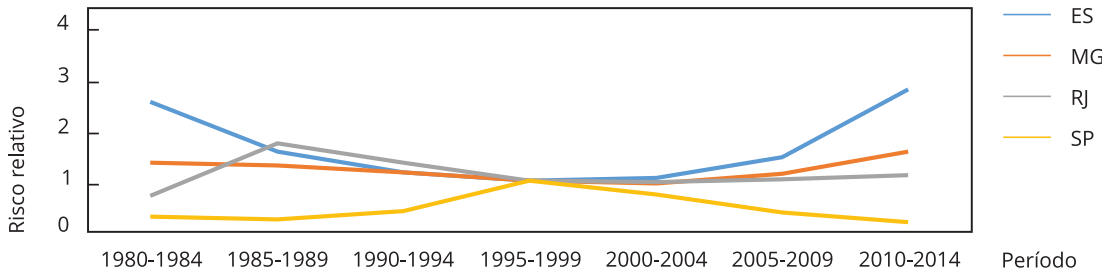

Estados: $\mathrm{AL}=$ Alagoas; $\mathrm{BA}=$ Bahia; $\mathrm{CE}=$ Ceará; $\mathrm{ES}=$ Espírito Santo; $\mathrm{MA}=$ Maranhão; $\mathrm{MG}=$ Minas Gerais; $\mathrm{PB}=\mathrm{Paraíba;}$ PE = Pernambuco; PI = Piauí; RJ = Rio de Janeiro; RN = Rio Grande do Norte; SE = Sergipe; SP = São Paulo.

(Figura 4). O Estado de São Paulo apresentou perfil inverso, com efeito de proteção para gerações mais jovens a partir da década de 1970 (Figura 4).

\section{Discussão}

Os principais achados deste estudo revelaram tendência ascendente da mortalidade por homicídios em homens em todos os estados da Região Nordeste e nos estados de Minas Gerais e Espírito Santo, na Região Sudeste. Em São Paulo e no Rio de Janeiro, houve descendência na mortalidade por homicídios no sexo masculino, com aumento da mortalidade até a terceira década de vida em todos os estados estudados e redução para os demais grupos etários. Nos estados da Região Nordeste, observou-se aumento do risco de morte nos anos 2000, com exceção da Bahia, Sergipe e Pernambuco. Em contrapartida, no mesmo período, na Região Sudeste, houve redução nos estados do Rio de Janeiro e São Paulo.

Os achados do presente estudo corroboram com as hipóteses de disseminação e interiorização da violência 8,9,10, pois, nos 35 anos estudados, houve tendência ascendente da mortalidade para todos os estados da Região Nordeste, com redução nos estados da Região Sudeste, com maior malha metropolitana, São Paulo e Rio de Janeiro.

No período de 2010-2014, a mortalidade por homicídios por 100 mil homens correspondeu a 91,97 óbitos na Região Nordeste e a 46,54 óbitos, na Região Sudeste, destacando-se os estados de Alagoas, Espírito Santo, Ceará e Sergipe como maiores taxas. Esses resultados colocam essas localidades entre as mais violentas do mundo, com coeficientes mais elevados se comparadas a outros países da América Latina, como El Salvador $(60,0)$, Jamaica $(56,0)$ e Venezuela $(53,7) 24$. Além disso, destaca-se 
que, nessas regiões, não se registram disputas territoriais ou guerra civil, sinalizando que a mortalidade violenta observada indica uma tragédia humana brasileira 6,8,25,26,27.

Neste estudo, verificou-se maior risco de óbito na segunda e terceira décadas de vida, em todos os estados das Regiões Nordeste e Sudeste, com aumento expressivo a partir dos 15-19 anos, e redução importante na quinta e sexta décadas de vida, seguindo a curva idade crime observada em outros estudos, corroborando, dessa maneira, com a teoria interacional 17,18. As localidades com coortes jovens largas (em número absoluto e relativo) apresentam maior incidência de atos criminosos e violência letal, devido ao processo denominado descontinuidade de coorte, que ocorre pelas flutuações no tamanho da coorte 19,20,2122,28,29,30,31.

Além disso, é importante destacar que o grau de vulnerabilidade de envolvimento com atos criminosos, como vítimas ou perpetradores, dos jovens brasileiros do sexo masculino apresenta diferenciais importantes de acordo com outras características, como raça/cor e classe social. Os jovens negros, moradores das periferias das cidades, apresentam maior risco de sofrerem agressão letal, devido à violência estrutural dos territórios onde residem 4,10,11,18,32. A estratificação da mortalidade por raça/cor apresenta disparidades importantes, que ajudam a analisar a mortalidade violenta dos jovens brasileiros, no período de 2005 a 2015, enquanto os homicídios reduziram 12,2\% entre indivíduos não negros; entre os negros, aumentaram 18,2\% 7. O caso de Alagoas merece destaque, pois, ao mesmo tempo que é o estado com maior chance de assassinato para um homem negro, é a localidade com menor risco de um homem branco sofrer violência letal 7 .

Os territórios com grande vulnerabilidade social concentram considerável proporção de população afrodescendente e têm sido denominados como "territórios de não Estado Democrático de Direito”, devido à ausência de políticas públicas que garantam direitos fundamentais 11,33. Nessas localidades, observam-se altas taxas de mortalidade por homicídios, perpetradas em disputas pelo controle do comércio ilegal de drogas, grupos de extermínio ou milícias e pelas Forças de Segurança do Estado que, em ações, constantemente, desrespeitam os direitos humanos, por meio do uso da força e de práticas letais, sem sofrer efetiva fiscalização do Ministério Público. Essa realidade que tem contribuído para elevadas taxas de mortalidade por homicídios em jovens, na faixa etária de 15 a 29 anos, sendo denominada, por alguns autores, como extermínio da população jovem, sobretudo nas periferias das grandes cidades e de raça/cor preta e parda 1,11,25,32,33,34. Nessa direção, estudo realizado para avaliar os diferencias na mortalidade jovem, com base no Censo de 2010, evidenciou que domicílios chefiados por mulheres, pessoas da raça/cor negra ou com ambas características apresentam maior chance de relatarem mortalidade de jovens entre 15 a 29 anos, realidade que se mantém quando se ajustam os dados para condição socioeconômica do domicílio 35 .

Alagoas, Ceará, Maranhão, Paraíba, Piauí e Rio Grande do Norte mostraram elevação do risco de morte em relação ao período de referência (1995-1999) e tendência ascendente. Sergipe e a Região Nordeste, apesar da redução do risco de morte nos períodos analisados em relação a 1995-1999, apresentaram evolução temporal ascendente, perfil semelhante na tendência de mortalidade foi verificado na Bahia, em que o risco de morte por período não foi estatisticamente significativo. Na Região Sudeste, em especial nos estados do Rio de Janeiro e de São Paulo, verificou-se redução do risco de morte nos anos 2000 com tendência descendente, e diminuição do risco de óbito por homicídio no último quinquênio no Estado do Espírito Santo e em Minas Gerais. Novamente, aponta-se o fortalecimento da hipótese do processo de disseminação e interiorização da violência que ocorreu no Brasil na virada para o século XXI. São Paulo e Rio de Janeiro, que, nas décadas anteriores apresentavam os maiores coeficientes de mortalidade por esse grupo de causas, nos anos 2000, mostram taxas inferiores aos demais estados 9,10 .

A redução no risco de óbito observada nos anos 2000, em Pernambuco, Rio de Janeiro e São Paulo, deve ser analisada com cautela, pois a evolução temporal da mortalidade pode sofrer efeito de período, no que se refere à qualidade da informação 14,15,16. Nesse sentido, no período de 2006 a 2009, no Município do Rio de Janeiro, a redução de 28,7\% na mortalidade por homicídios foi acompanhada por importante aumento de óbitos por ECI (62,5\%), e, após aplicação de técnicas de correção, verificou-se que essa redução foi de 3,6\% 36. No presente estudo, observou-se que, no Estado de São Paulo, a redução do risco de óbito por homicídios foi acompanhada da diminuição do risco de óbitos classificados como ECI, enquanto que, em Pernambuco, a diminuição do risco de morte por homicídios foi acom- 
panhada pela elevação da probabilidade de óbito por ECI em todos os quinquênios, perfil semelhante ocorreu no Rio de Janeiro, no período de 2005 a 2014.

Os achados deste estudo sinalizam que a redução do risco de morte por homicídios observada em São Paulo não está correlacionada ao efeito de período da qualidade da informação (óbitos por ECI), pois houve redução da probabilidade de morte para ambas as classificações ao mesmo tempo. Estudos têm relacionado a redução dos homicídios no Estado e Município de São Paulo à estrutura etária da população, a investimentos em educação e cultura, à taxa de encarceramento e a armas apreendidas 37,38 . No entanto, é importante destacar que todas essas variáveis perderam significância quando os modelos foram ajustados para taxa de desemprego e redução de jovens na população ${ }^{3}$. A diminuição de jovens na população, em meados da década de 1990 e início dos anos 2000, foi responsável por 7,14\% da queda nas taxas de homicídios no Estado de São Paulo 39 , sinalizando para o efeito da descontinuidade de coorte na mortalidade por homicídios nesse estado.

O risco de óbito por homicídio segundo coorte evidenciou aumento progressivo para as gerações mais jovens, a partir dos homens nascidos na década de 1960, em todos os estados da Região Nordeste, com exceção de Pernambuco, em que as gerações nascidas a partir de 1975-1979 mostraram aumento do risco de morte, o mesmo foi observado no Rio de Janeiro, em Minas Gerais e no Espírito Santo. Em São Paulo, verificou-se perfil inverso, com redução do risco para gerações mais jovens.

Os achados relativos às regiões Nordeste e Sudeste, com exceção de São Paulo, são semelhantes aos resultados dos estudos realizados nos Estados Unidos 21,29, que evidenciaram maior risco de morte por homicídio em homens nascidos a partir da década de 1960, com maior pico de incidência na década de 1980, com menor risco nos anos 2000. Esses resultados foram correlacionados ao aumento de jovens na população com outros fatores criminogênicos, como a elevação da prevalência de consumo de cocaína, crack e circulação de armas de fogo, sobretudo nas áreas de maior vulnerabilidade social. Destaca-se que a chance de óbito por homicídio em homens jovens, nos períodos analisados, elevou-se quando os modelos APC foram ajustados para variáveis individuais, como participação em gangues (OR = 2,27; IC95\%: 1,59-3,53), possuir arma de fogo (OR = 3,52; IC95\%: 2,55-4,85) e ser usuário de drogas pesadas (OR = 2,79; IC95\%: 1,91-4,06) 29 .

$\mathrm{O}$ aumento do risco de morte para as coortes mais jovens nos estados das regiões Nordeste e Sudeste, com exceção de São Paulo, pode estar relacionado ao aumento absoluto e relativo do número de adolescentes e jovens na estrutura etária populacional dessas localidades 19,20,21,22,28,29,30, devido ao alargamento da base da pirâmide etária, nos períodos de 1950-1965 e 1975-1985, e à redução significativa na taxa de mortalidade infantil 36 . Isso promoveu a denominada descontinuidade de coorte, em que os integrantes de largas coortes terão menos oportunidade de vida em relação ao mercado de trabalho e à educação de qualidade, alimentando o ciclo da violência, devido à restrição material e anomia social 19,20,21,22,23,28,29,30,37. Assim, esses indivíduos são expostos à maior competição pelo mercado de trabalho na idade adulta e à menor possibilidade de seguridade econômica, sujeitando-os a alto nível de desvio de conduta, por dificuldade em alcançar as metas sociais de sucesso e sobrevivência 19,20, associada à ineficiência do Estado em garantir os direitos sociais básicos 18,20,21,22,40,41,42. Estudo desenvolvido por Pampel \& Gardner 43 evidenciou que a correlação entre estrutura etária da população e taxa de criminalidade foi atenuada em países com instituições de proteção social mais desenvolvidas e fortes.

O aumento absoluto e relativo de jovens na população não é causa suficiente para elevação da criminalidade e violência letal, outros fatores criminogênicos, como desigualdade social, educação de baixa qualidade, evasão escolar de pré-adolescentes e adolescentes, tráfico de drogas, alta circulação de armas de fogo, consumo abusivo de álcool e outras drogas, violência policial, entre outros, podem alterar a correlação entre tamanho relativo das coortes jovens e a violência 19,20,21,22,28,29,30,40,41.

O cenário apresentado pelo Estado de Pernambuco no risco de morte, inclusive nas coortes mais velhas, em relação à coorte de 1950-1954, diferindo dos demais estados que apresentaram efeito de proteção, pode correlacionar-se com o fato de esse estado apresentar altas taxas de mortalidade desde o início da série histórica, portanto, outros fatores, como a privação econômica e o baixo controle social, podem contribuir para suavizar a relação entre a idade e as taxas de homicídio, e aumentar o risco de violência letal para toda a população independente da idade 22,29. No Estado de São Paulo, a redução do risco de homicídios nas coortes mais jovens pode se relacionar ao efeito do período 
promovido pela redução da proporção de jovens na população e ao efeito de período de melhorias na condição socioeconômica (redução na taxa de desemprego), ampliação do acesso à educação (aumento de jovens matriculados no Ensino Médio) e às medidas de Segurança Pública implementadas nos anos $200037,38,39$.

Os resultados do presente estudo devem ser analisados com cautela, pois podem ser influenciados pela qualidade da informação, pois, ao longo dos 35 anos analisados, houve melhorias significativas na cobertura e certificação dos óbitos, o que pode gerar efeito de período na tendência de mortalidade por causa específica 14,15,16 (Sistema de Informações sobre Mortalidade. http://www2.datasus. gov.br/DATASUS/index.php?area=0205, acessado em 28/Nov/2018). Desse modo, a magnitude da mortalidade por homicídios nas localidades analisadas pode estar subestimada, devido à grande proporção de registros de óbitos classificados como ECI, apresentando tendência ascendente em muitos estados, como a Bahia que apresentou incremento superior a 20\% na mortalidade por ECI. A grande proporção de óbitos classificados nesse grupo pode mascarar reduções na mortalidade por homicídio, que figuram erroneamente como ECI 44. Por esse motivo, no presente trabalho, analisou-se o efeito do período dos óbitos classificados como ECI.

Outra limitação refere-se aos modelos APC, pois não há consenso na literatura sobre o melhor método para corrigir o problema de identificabilidade do modelo completo, no entanto, neste estudo, os modelos foram estimados por meio de funções estimáveis, metodologia mais recomendada pelos estudos que comparam os métodos da estatística clássica 14,15,16. Autores como O’Brien \& Stockard 19 propõem a realização de análises desagregadas por raça/cor e por famílias monoparentais, por exemplo, tendo em vista que tais características e circunstâncias tendem a elevar o risco de morrer por homicídio, o que deve ser realizado em estudos posteriores.

\section{Conclusão}

A avaliação da evolução da mortalidade por homicídios em pessoas do sexo masculino, nas regiões Nordeste e Sudeste, retratou o processo de disseminação da violência ocorrido no Brasil nos anos 2000, assim como maior risco de morte para adultos jovens, nascidos em gerações a partir da década de 1970, com exceção do Estado de São Paulo. Demonstrou, também, o grave quadro de mortalidade violenta da população jovem brasileira, que tem sido denunciado por organizações não governamentais, movimentos sociais e organizações de direitos humanos, como o genocídio dos jovens. As políticas sociais implementadas a partir dos anos 2000, que visaram diminuir as desigualdades, não foram acompanhadas por redução na violência letal. Realidade que pode agravar-se com a política de ajuste fiscal implementada pela Emenda Constitucional 95 45, pois resultará em grande contingente de jovens sem acesso aos direitos sociais básicos, ampliando o risco de envolvimento em atos de criminalidade e vitimização por homicídio. 


\section{Colaboradores}

L. F. Borges contribuiu com a análise dos dados. E. R. Souza contribuiu com a interpretação dos dados, redação e revisão crítica relevante do conteúdo intelectual. A. P. Ribeiro e G. W. S. Silva contribuíram com a concepção do projeto e revisão crítica relevante do conteúdo intelectual. C. M. F. P. Silva contribuiu com a análise crítica dos resultados e revisão crítica relevante do conteúdo intelectual. J. Santos contribuiu com a concepção e projeto e revisão crítica relevante do conteúdo intelectual. K. C. Meira contribuiu com a concepção e projeto e análise e interpretação dos dados. Todos os autores aprovaram a versão final a ser publicada.

\section{Informações adicionais}

ORCID: Laiane Felix Borges (0000-0001-53582591); Edinilsa Ramos de Souza (0000-0003-09034525); Adalgisa Peixoto Ribeiro (0000-0001-94158068); Glauber Weder dos Santos Silva (0000-00020570-1944); Cosme Marcelo Furtado Passos da Silva (0000-0001-7789-1671); Juliano dos Santos (0000-0001-9961-3576); Karina Cardoso Meira (0000-0002-1722-5703).

\section{Agradecimentos}

À Coordenação de Aperfeiçoamento de Pessoal de Nível Superior, pelo fomento a esta pesquisa (Código de financiamento 001).

\section{Referências}

1. Cruz-Neto O, Minayo MCS. Extermínio: violentação e banalização da vida. Cad Saúde Pública 1994; 10 Suppl 1:199-212.

2. Souza ER, Lima MLC, Bezerra EAD. Homicides in Brazil: evolution and impacts. In: Lovisi GM, Mari JJ, Valencia ES, editors. The psychological impact of living under violence. New York: Nova Science Publishers; 2010. p. 1-14.

3. Melo ACM, Silva GDM, Garcia LP. Mortalidade de homens jovens por agressões no Brasil, 2010-2014: estudo ecológico. Cad Saúde Pública 2017; 33:e00168316.

4. Peres MFT, Vicentin D, Nery M, Lima RS, Souza ER, Cerda M, et al. Queda dos homicídios em São Paulo, Brasil: uma análise descritiva. Rev Panam Salud Pública 2011; 29:17-26.

5. Szwarcwald CL, Castilho EA. Mortalidade por armas de fogo no Estado do Rio de Janeiro, Brasil: uma análise espacial. Rev Panam Salud Pública 1998; 4:161-70.

6. Cerqueira D, Lima RS, Bueno S, Valencia LI, Hanashiro O, Machado PHG, et al. Atlas da violência 2017. http://www.ipea.gov.br/por tal/index.php?option $=$ com_content \&view $=$ article $\& \mathrm{id}=30411$ (acessado em 27/Dez/ 2018).

7. Cerqueira D, coordenador. Atlas da violência 2018. http://www.ipea.gov.br/portal/ index.php?option $=$ com_content $\&$ view $=$ arti cle\&id=33410\&Itemid $=432$ (acessado em 27/ Dez/2018).

8. Steeves GM, Petterini FC, Moura GV. The interiorization of Brazilian violence, policing, and economic growth. Economia 2015; 16:359-75.

9. Waiselfisz JJ. Mapa da violência 2016: homicídios por armas de fogo. https://www.mapadaviolencia.org.br/pdf2016/Mapa2016_armas_ web.pdf (acessado em 27/Dez/2018).

10. Andrade LT, Diniz AMA. A reorganização espacial dos homicídios no Brasil e a tese da interiorização. Rev Bras Estud Popul 2013; 30 Suppl:S171-91.

11. Zaluar A. Democratização inacabada: fracasso da segurança pública. Estud Av 2007; 21:3149.

12. Wintemute GJ. The epidemiology of firearm violence in the twenty-first century United States. Annu Rev Public Health 2015; 36:5-19.

13. Tavares R, Catalan VDB, Romano PMM, Melo EM. Homicídios e vulnerabilidade social. Ciênc Saúde Colet 2016; 21:923-34.

14. Holford TR. The estimation of age, period and cohort effects for vital rates. Biometrics 1983; 39:311-24.

15. Robertson B, Boyle P. Age period-cohort analysis of chronic disease rates. I: Modelling approach. Stat Med 1998; 17:1305-23.

16. Holford TR. Approaches to fitting age-period cohort models for the Lexis diagram. Stat Med 2006; 26:3018-45. 
17. Cerqueira D, Lobão W. Determinantes da criminalidade: arcabouços teóricos e resultados empíricos. Dados 2004; 47:233-69.

18. Cerqueira D, Moura RL. O efeito das oportunidades no mercado de trabalho sobre as taxas de homicídios no Brasil. In: Corseuil CH, Botelho RU, organizadores. Desafios à trajetória profissional dos jovens brasileiros. Rio de Janeiro: Instituto de Pesquisa Econômica Aplicada; 2014. p. 267-90.

19. O'Brien RM, Stockard J. Variations in agespecific homicide death rates: a cohort explanation for changes in the age distribution of homicide deaths. Soc Sci Res 2002; 31:124-50.

20. O’Brien RM. Relative cohort size and agespecific crime rates: an age-period-cohort size model. Criminology1989; 27:57-78.

21. Shahpar C, Li G. Homicide mortality in the United States, 1935-1994: age, period, and cohort effects. Am J Epidemiol 1999; 150:121322.

22. Philips JA. The relationship between age structure and homicide rates in the United States, 1970 to 1999. J Res Crime Delinq 2006; 43:230-60.

23. Cerqueira D, Moura RL. Custo da juventude perdida no Brasil. In: Corseuil $\mathrm{CH}$, Botelho $\mathrm{RU}$, organizadores. Desafios à trajetória profissional dos jovens brasileiros. Rio de Janeiro: Instituto de Pesquisa Econômica Aplicada; 2014. p. 291-320.

24. Muggah R, Tobón KA. Citizen security in Latin America: facts and figures. Rio de Janeiro: Igarapé Institute; 2018. (Strategic Paper, 33).

25. Reichenheim ME, Souza ER, Moraes CL, Mello-Jorge MHP, Silva CMFP, Minayo MCS. Violence and injuries in Brazil: the effect, progress made, and challenges ahead. Lancet 2011; 377:1962-75.

26. Souza ER, Melo AN, Silva JG, Franco SA, Alazraqui M, González-Pérez GJ. Estudo multicêntrico da mortalidade por homicídios em países da América Latina. Ciênc Saúde Colet 2012; 17:3183-93.

27. Gawryszewski VP, Sanhueza A, Martinez-Piedra R, Escamilla JA, Souza MFM. Homicídios na região das Américas: magnitude, distribuição e tendências, 1999-2009. Ciênc Saúde Colet 2012; 17:3171-82.

28. Araujo Jr. AF. Decomposição dos efeitos idade, período e coorte de taxas de homicídios: uma análise por estados - 1981-1996. Economia \& Gestão 2002; 2:91-108.

29. Fabio A, Loeber R, Balasubramani GK, Roth J, $\mathrm{Fu}$ W, Farrington DP. Why some generations are more violent than others: assessment of age, period, and cohort effects. Am J Epidemiol 2006; 164:151-60.

30. Nivette AE. Cross-national predictors of crime: a meta-analysis. Homicide Stud 2011; 15:103-31.

31. Cole JH, Gramajo AM. Homicide rates in a cross-section of countries: evidence and interpretations. Popul Dev Rev 2009; 35:749-77.
32. Cerqueira D, Coelho DSC. Democracia racial e homicídios de jovens negros na cidade partida. Brasília: Instituto de Pesquisa Econômica Aplicada; 2017. (Texto para Discussão, 2.267).

33. Peres MFT, Cardia N, Mesquita Neto P, Santos PC, Adorno S. Homicídios, desenvolvimento socioeconômico e violência policial no Município de São Paulo, Brasil. Rev Panam Salud Pública 2008; 23:268-76.

34. Lemos FCS, Aquime RHS, Franco ACF, Piani PPF. O extermínio de jovens negros pobres no Brasil: práticas biopolíticas em questão. Pesqui Prát Psicossociais 2017; 12:164-76.

35. Pereira FNA, Queiroz BL. Diferenciais de mortalidade jovem no Brasil: a importância dos fatores socioeconômicos dos domicílios e das condições de vida nos municípios e estados brasileiros. Cad Saúde Pública 2016; 32:e00109315.

36. Cerqueira D. Mapa dos homicídios ocultos no Brasil. Brasília: Instituto de Pesquisa Econômica Aplicada; 2013. (Texto para Discussão, 1.848).

37. Peres MFT, Almeida JF, Vincentin D, Ruotti C, Nery MB, Cerda M, et al. Evolução dos homicídios e indicadores de segurança pública no Município de São Paulo entre 1996 a 2008: um estudo ecológico de séries temporais. Ciênc Saúde Colet 2012; 17:3249-57.

38. Peres MFT, Almeida JF, Vincentin D, Ruotti C, Nery MB, Cerda M, et al. Queda dos homicídios no Município de São Paulo: uma análise exploratória de possíveis condicionantes. Rev Bras Epidemiol 2011; 14:709-21.

39. Mello JMP, Schneider A. Mudança demográfica e a dinâmica dos homicídios no Estado de São Paulo. São Paulo Perspect 2007; 21:19-30.

40. Bercovich A, Madeira F. Descontinuidades demográficas no Brasil e no Estado de São Paulo. In: Anais do VII Encontro Nacional de Estudos Populacionais. v. 2. Belo Horizonte: Associação Brasileira de Estudos Populacionais; 1990; p. 595-632.

41. Easterlin RA. Birth and fortune: the impact of numbers on personal welfare. Chicago: University of Chicago Press; 1987.

42. Muniz JO. As descontinuidades demográficas exercem efeito sobre o mercado de trabalho metropolitano dos jovens. Rev Bras Estud Popul 2002; 19:67-98.

43. Pampel F, Gardner R. Age structure, socialpolitical institutions, and national homicide rates. Eur Sociol Rev 1995; 11:243-60.

44. Cerqueira D. Mortes violentas não esclarecidas e impunidade no Rio de Janeiro. Economia Aplicada 2012; 16:201-35.

45. Brasil. Emenda Constitucional no 95, de 15 de dezembro de 2016. Altera o Ato das Disposições Constitucionais Transitórias, para instituir o Novo Regime Fiscal, e dá outras providências. Diário Oficial da União 2016; 16 dez. 


\section{Abstract}

The objective was to assess the effect of age, period, and birth cohort on the time trend in mortality from homicides in men in the states of the Northeast and Southeast regions of Brazil from 1980 to 2014. This was an ecological time trend study. PCA models were estimated using estimable functions in inference of the parameters. Mortality and population data were obtained from the Brazilian Health Informatics Department. State-by-state mortality rates from homicide were standardized by the direct method, with the world population as the standard, as proposed by the World Health Organization. The Northeast region recorded 317,711 deaths from homicides and the Southeast 544, 640 deaths, corresponding, respectively, corresponding to mean standardized rates of 58.68 and 64.68 deaths per 100,000 men. The highest mean standardized mortality rates were observed in the states of Alagoas (157.74 deaths) and Pernambuco (109.58 deaths). All the states showed an increase in mortality up to the third decade of life, with a progressive reduction in the other age brackets. There was an upward trend in all the states of the Northeast and in Minas Gerais and Espirito Santo in the Southeast, while in the other states there was a downward trend. All the states showed an increase in the risk of death in the younger age brackets, except for the state of São Paulo, which showed the inverse profile. The current study's findings may correlate with the process of discontinuity in the cohort, in which members of wide cohorts found less opportunity for access to employment, income, and education, thus increasing the risk of involvement in crime and death from homicide.

Homicide; Men; Age Effect; Period Effect; Cohort Effect

\section{Resumen}

El objetivo fue evaluar el efecto de la edad, período $y$ cohorte de nacimiento, en la evolución temporal de la mortalidad por homicidios en hombres, en los estados de las regiones Nordeste y Sudeste, Brasil, entre el período de 1980 a 2014. Se realizó un estudio ecológico de tendencia temporal. Los modelos APC se estimaron usando funciones estimables en la inferencia de los parámetros. Los datos de mortalidad y los datos poblacionales se obtuvieron mediante el Departamento de Informática del Sistema Único de Salud. Las tasas de mortalidad por homicidio, según los diferentes estados, se estandarizaron mediante método directo, considerando como población patrón la mundial, propuesta por la Organización Mundial de la Salud. La Región Nordeste presentó 317.711 óbitos por homicidios y el Sudeste 544.640 óbitos, correspondiendo a una tasa media estandarizada por 100.000 hombres de 58,68 óbitos y 64,68 óbitos, respectivamente. Las mayores tasas de mortalidad medias estandarizadas se observaron en los estados de Alagoas (157,74 óbitos) y Pernambuco (109,58 óbitos). En todos los estados se observó un aumento de la mortalidad hasta la tercera década de vida, con una reducción progresiva para las demás franjas etarias. Se verificó una tendencia ascendente para todos los estados de la Región Nordeste, Minas Gerais y Espírito Santo, en los demás estados hubo un descenso de las tasas. En todos los estados, se observó un aumento del riesgo de óbito en las generaciones más jóvenes, con excepción del Estado de São Paulo, que mostró un perfil inverso. Los hallazgos del presente estudio pueden correlacionarse con el proceso de discontinuidad de cohorte, en donde integrantes de cohortes más grandes encuentran una menor oportunidad de acceso al empleo, renta y educación, así como un aumento el riesgo de implicación en la criminalidad y muerte por homicidios.

Homicidio; Hombres; Efecto Edad; Efecto Periodo; Efecto de Cohortes

Recebido em 16/Jan/2019

Versão final reapresentada em 27/Mai/2019

Aprovado em 06/Jun/2019 